\title{
Calculation of Gamma Functions to High Accuracy
}

\author{
By M. E. Sherry and S. Fulda*
}

The two constants, $\Gamma\left(\frac{1}{3}\right)$ and $\Gamma\left(\frac{2}{3}\right)$ have been calculated to 35 decimal places on the Cambridge Computer by means of an interpretive routine that treats floating-point numbers of 37 significant digits $[1,2]$.

Stirling's asymptotic expansion for $\ln \Gamma(x)$ can be written

$$
\ln \Gamma(x) \sim\left(x-\frac{1}{2}\right) \ln x-x+\frac{1}{2} \ln 2 \pi+\sum_{r=1}^{\infty} \frac{C_{r}}{x^{2 r-1}}
$$

With the remainder of the series made very small, the accuracy of $\ln \Gamma(x)$ depends primarily on the accuracy of $\ln 2 \pi, \ln x$, and the $C_{r}$. Uhler $[3,4]$ has published the $\ln x$ for all primes through 101 , as well as the $\ln \pi$, to more than 100 significant figures. Uhler has also calculated the $C_{r}$ to over 100 significant figures [5].

The recursion formula for the Gamma function

$$
\Gamma(x+1)=x \Gamma(x)
$$

can be extended to

$$
\Gamma(x+n)=(x)_{n} \Gamma(x)
$$

where $(x)_{n}$ is the Pochhammer-Barnes symbol

$$
(x)_{n}=(x+n-1)(x+n-2) \cdots(x+1) x .
$$

If we take the logarithm of Eq. (3),

$$
\ln \Gamma(x+n)=\ln \Gamma(x)+\sum_{j=0}^{n-1} \ln (x+j) .
$$

Substituting Eq. (1) into Eq. (5) gives, after solving for $\ln \Gamma(x)$,

$$
\ln \Gamma(x) \sim \lambda(x)+\sum_{r=1}^{\infty} \frac{C_{r}}{(x+n)^{2 r-1}},
$$

where

(7) $\lambda(x)=\left(x+n-\frac{1}{2}\right) \ln (x+n)-(x+n)+\frac{1}{2} \ln 2 \pi-\sum_{j=0}^{n-1} \ln (x+j)$.

The term $\lambda(x)$ is calculated quite easily by hand, and it is not difficult to calculate $\ln \Gamma(x)$ from Eq. (6) with a digital computer.

To calculate $\Gamma(x)$, let

$$
\Gamma(x)=e^{\psi} e^{\ln \Gamma(x)-\psi}
$$

Received December 31, 1958; revised May 4, 1959.

* S. Fulda, Bell Telephone Laboratories, Murray Hill, New Jersey, co-authored this paper while on temporary duty at the Air Force Cambridge Research Center. 
where

$$
\psi= \pm 0.1 n
$$

is the exponent of $e$ nearest in value to $\ln \Gamma(x)$. Values of $e^{ \pm 0.1 n}, n$ being any integer from 0 to 10, have been tabulated by Van Orstrand [6].

(10) Since

$$
|\ln \Gamma(x)-\psi| \leqq 0.05
$$

it will always be possible to calculate $e^{\ln \Gamma(x)}$ by means of the exponential power series expansion using very few terms.

These techniques led to the following values:

$$
\begin{aligned}
\Gamma\left(\frac{1}{3}\right) & =2.6789385347077476336556929409746776 \\
\Gamma\left(\frac{2}{3}\right) & =1.3541179394264004169452880281545138 \\
\ln \Gamma\left(\frac{1}{3}\right) & =.98542064692776706918717403697796139 \\
\ln \Gamma\left(\frac{2}{3}\right) & =.30315027514752356867586281737201104 .
\end{aligned}
$$

It was possible to examine round-off and truncation errors at each step in the calculation. The final relative error was less than $\pm 3 \times 10^{-35}$ in each case.

As a final independent check, the values of $\Gamma\left(\frac{1}{3}\right)$ and $\Gamma\left(\frac{2}{3}\right)$ were put into

$$
\Gamma\left(\frac{1}{3}\right) \Gamma\left(\frac{2}{3}\right)=\frac{2 \pi}{\sqrt{3}} .
$$

The error analysis was consistent with this identity.

Computer \& Mathematical Sciences Laboratory,

Air Force Cambridge Research Center,

Bedford, Massachusetts

1. B. ZONDEK, "The values of $\Gamma\left(\frac{1}{3}\right)$ and $\Gamma\left(\frac{2}{3}\right)$ and their logarithms accurate to 28 decimals," $M T A C$, v. 9,1955 , p. 24-25.

2. Air Force Cambridge Research Center Report, Cambridge Computer Interpretive Routine for Quadruple Precision Numbers, Series 3, TN-59-155, 1959.

3. H. S. UhLER, "Natural logarithms of small primary numbers," Proc., Nat. Acad. Sci., v. 29,1943, p. 319-325.

4. H. S. Uhler, "Log $\pi$ and other basic constants," Proc., Nat. Acad. Sci., v. 24, 1938, p. 23-30.

5. H. S. Uhler, "The coefficients of Stirling's series for $\log \Gamma(x)$," Proc., Nat. Acad. Sci., v. 28,1942, p. $59-62$.

6. C. E. VAN Orstrand, "Tables of the exponential function and of the circular sine and cosine to radian argument," Mem., Nat. Acad. Sci., v. 14: 5, 1921 (Tables IV and V). 